\title{
Abdominal cerclage after failed transvaginal cervical cerclage
}

\author{
Anwar Moria • Nouf Aljaji • Louise Miner • \\ Togas Tulandi
}

Received: 25 May 2011 / Accepted: 15 June 2011 /Published online: 1 July 2011

(C) Springer-Verlag 2011

\begin{abstract}
This study aimed to evaluate the efficacy of abdominal cerclage by laparotomy and by laparoscopy among women who had failed transvaginal cerclage. We evaluated all women with cervical insufficiency that underwent abdominal cerclage between the years 2000 and 2010. Of a total of 20 patients, 12 patients underwent abdominal cerclage by laparoscopy and 8 others by laparotomy. The procedure was done in the pregnant state in four patients in the laparoscopy group and in all patients in the laparotomy group. There was no significant difference in the operative time between the laparoscopy and the laparotomy group. The median duration of hospitalization in the laparoscopy group was $6 \mathrm{~h}$ and in the laparotomy group was $96 \mathrm{~h}$. Of 18 pregnancies, 16 resulted in a live birth $(88.9 \%)$. Abdominal cerclage in women who have failed a transvaginal cervical cerclage is associated with a high live birth rate.
\end{abstract}

Keywords Cervical incompetence - Cervical insufficiency Cervical cerclage $\cdot$ Abdominal cerclage $\cdot$ Cervicoisthmic cerclage

\section{Background}

Cervical cerclage has been used to prevent preterm birth in high-risk women with cervical insufficiency, with a short

A. Moria $\cdot$ N. Aljaji $\cdot$ L. Miner $\cdot$ T. Tulandi

Department of Obstetrics and Gynecology, McGill University,

Montreal, QC, Canada

T. Tulandi $(\square)$

687 Pine Ave. West,

Montreal, Quebec H3A 1A1, Canada

e-mail: togas.tulandi@mcgill.ca and/or dilated cervix, and in those with a high risk of preterm delivery. It is usually applied through the vagina after confirmation of fetal viability. Abdominal cervical cerclage was first described in 1965 [1]. This approach is different from the traditional vaginal McDonald [2] or Shirodkar [3] cerclage. The cerclage is placed at the cervicoisthmic junction through an abdominal approach after reflecting the bladder away. The advantages of abdominal cerclage include more proximal placement of the suture providing a better support for the pregnancy, decreased risk of suture migration, absence of a foreign body in the vagina that could promote infection, and the ability to leave the suture in situ for future pregnancies. In a review, Zaveri et al. concluded that women with a failed transvaginal cervical cerclage in a previous pregnancy had a lower risk of perinatal death or delivery of less than 24 weeks if an abdominal cerclage was placed instead of repeat transvaginal cerclage [4]. The purpose of our study was to evaluate the efficacy of abdominal cerclage by laparotomy and by laparoscopy among women with cervical insufficiency who had failed transvaginal cervical cerclage.

\section{Materials and methods}

We evaluated the charts of all women who underwent abdominal cervical cerclage from the year 2000 to 2010 at the Sir Mortimer B. Davis, Jewish General Hospital. The indication of abdominal cerclage was at least one failed transvaginal cervical cerclage. Information was retrieved from the hospital's medical file and crosschecked with the clinic charts. Cases of emergency transvaginal cervical cerclage were excluded. The research ethics office of the hospital approved the study. 


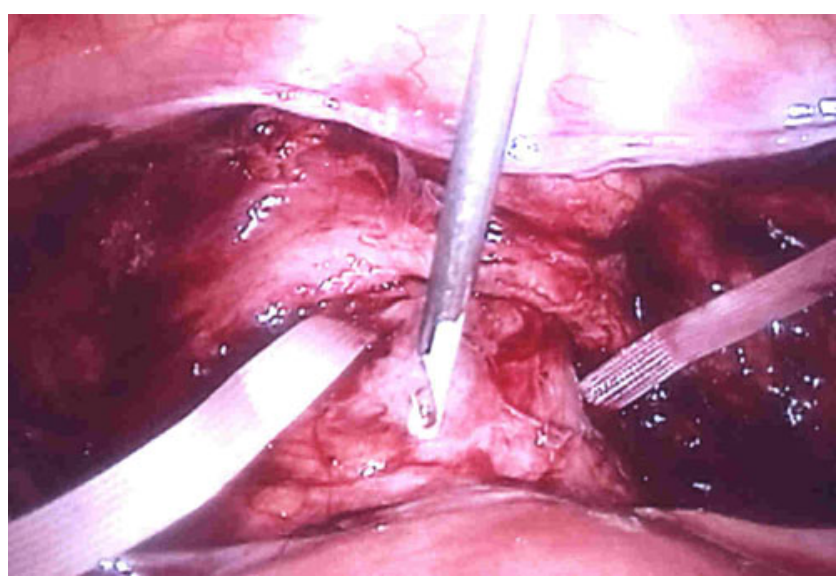

Fig. 1 The Mersilene tape has been placed surrounding the cervix. Note the opening at the tip of the suturing device

Between the years 2000 and 2004, abdominal cerclage was conducted by laparotomy around 12 weeks of gestation after confirmation of fetal heart activity and was performed by the patient's treating obstetrician. As of 2005, all abdominal cerclage was performed by the senior author (TT) through laparoscopy. In both techniques, we started by dissecting the bladder peritoneum off the cervix and we used 5-mm Mersilene tape (Ethicon Inc., Peterborough, Ontario, Canada) for the cerclage. In the laparotomy technique, the suture was inserted through the avascular area of the paracervical tissue medial to the uterine vessels and at the level of the uterine isthmus.

The laparoscopic abdominal cerclage was performed as we previously described [5]. In short, we used a disposable EndoClose suturing device (Tyco Health Care, Gasport, UK) piercing the body of the cervix medial to the uterine vessels without dissecting the ureter and the vessels. The tapered end of the tape was inserted into the distal opening of the device and retracted anteriorly. The procedure was repeated on the opposite site and the knot was tied

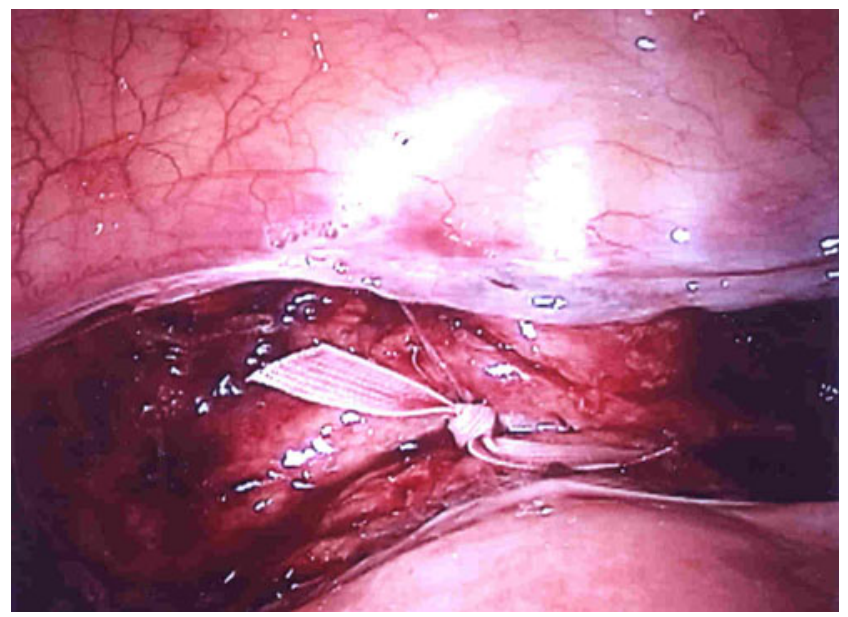

Fig. 2 Completion of the abdominal cerclage with the knot on the anterior wall of the cervix
Table 1 Profiles of 20 women who had failed transvaginal cerclage and subsequently underwent abdominal cervical cerclage

\begin{tabular}{lll}
\hline & Laparoscopy & Laparotomy \\
\hline Number & 12 & 8 \\
Age (years) & $34.4 \pm 1.3$ & $34.2 \pm 0.8$ \\
BMI & $24.9 \pm 1.6$ & $23.3 \pm 2.4$ \\
Operative time (min) & $64.2 \pm 6.1$ & $66.1 \pm 10.0$ \\
\hline
\end{tabular}

$B M I$ body mass index

anteriorly (Figs. 1 and 2). We evaluated operative time, intra- and postoperative complications, the duration of hospitalization, and outcome of pregnancies.

\section{Statistical analysis}

We used Shapiro-Wilk test to evaluate the distribution of the data. Comparisons were analyzed using Student's $t$ test or Mann-Whitney $U$ test when appropriate. A $P$ value of less than 0.05 was considered significant.

\section{Findings}

Of a total of 20 patients, 12 patients underwent abdominal cerclage by laparoscopy and 8 others by laparotomy. The procedure was done in the pregnant state in four patients in the laparoscopy group and in all patients in the laparotomy group. The age and body mass index of both groups of patients were comparable (Table 1). We did not encounter any excessive bleeding during surgery in both groups of patients. There was no significant difference in the operative time between the laparoscopy and the laparotomy group. The median duration of hospitalization in the laparoscopy group was $6 \mathrm{~h}$ and in the laparotomy

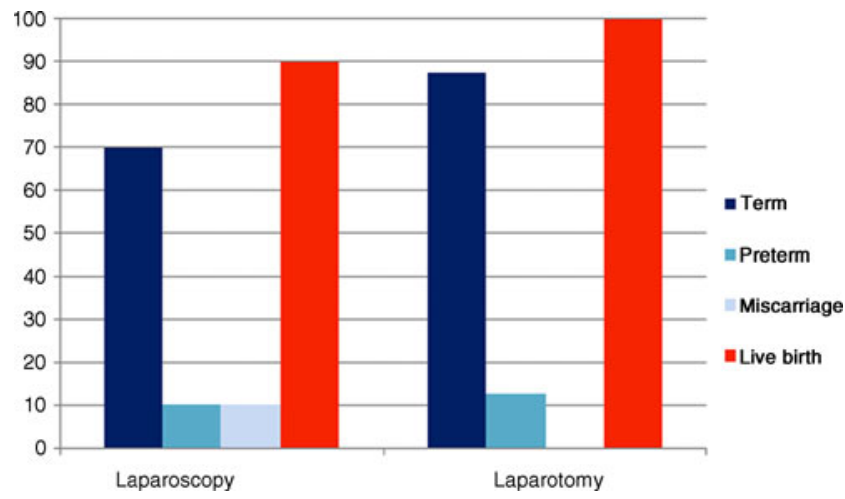

Fig. 3 Pregnancy outcome in 18 pregnancies among women who had failed transvaginal cerclage and subsequently underwent abdominal cervical cerclage 
group was $96 \mathrm{~h}$ (interquartile range $80-108)(P<0.0001$; 95\% CI, 66-114).

At the time of this writing, two patients in the laparoscopic group who recently underwent the procedure had not conceived and another was lost from follow-up leaving nine evaluable cases for obstetrical outcome. Seven patients had successful term pregnancy; one patient experienced a miscarriage at 14 weeks of gestation. She underwent curettage with the abdominal cerclage in situ, and in the subsequent pregnancy she delivered at 34 gestational weeks. Another patient had severe oligohydramnios at 19 gestational weeks and underwent laparoscopic removal of the cerclage. Of a total of eight patients in the laparotomy group, seven patients had a successful term pregnancy and another had premature delivery at 29 weeks of gestation. Overall, 16 of 18 pregnancies resulted in a live birth (88.9\%, Fig. 3).

There was no postoperative complication in the laparoscopy group. In the laparotomy group, one patient had wound infection and another had urinary tract infection in the immediate postoperative period.

\section{Discussion}

In our study, abdominal cervical cerclage resulted in $88.9 \%$ live birth rate. This is in agreement with a previous study in 2009 [6], where the fetal salvage rate was $89 \%$. They used criteria for a presumptive diagnosis of cervical insufficiency including failed transvaginal cerclage in $58 \%$ of cases. Conversion to laparotomy was needed in $10 \%$ of cases due to bleeding from the uterine vessels and impaired visibility [6].

In contrast, all of our patients had a history of failed transvaginal cerclage. Further, our laparoscopic technique was slightly different. Instead of using Prolene suture, we used 5-mm Mersilene tape [5]. We also utilized an EndoClose suturing device that was inserted medial to the uterine vessels piercing the cervical body. Unlike the conventional abdominal cerclage, dissection of the vessels and ureter was not needed simplifying the procedure. Indeed, none of the laparoscopic procedures were converted to laparotomy, and bleeding was minimal.

Although there is a clear obstetrical advantage of abdominal cerclage over transvaginal cervical cerclage in high-risk populations, abdominal cerclage has not been popular. This is due to the need for two surgical procedures; the first is to perform the cerclage and the second is for cesarean delivery. In addition, there has been a concern that if a miscarriage or fetal demise was to occur, a hysterotomy would have to be performed. However, today, abdominal cerclage can be performed by laparoscopy. In the case of a miscarriage, curettage can still be done with the cerclage in place. In the case of second trimester loss, the cerclage can be removed by laparoscopy precluding the need for hysterotomy [7].

Previous reports and ours demonstrated that one could perform abdominal cerclage before pregnancy. Indeed, surgery in the non-pregnant state is associated with decreased operative bleeding and no risk of miscarriage or rupture membrane. Moreover, it requires only one surgery in the pregnant state which is the cesarean delivery. Groom et al. in 2003 reported 19 women with preconceptional abdominal cerclage insertion and a fetal survival rate of $100 \%$ in women who were able to get pregnant [8]. This is compared favorably with our results in the women who underwent abdominal cerclage in the pregnant state where all of our cases subsequently had a live birth.

The advantages of laparoscopic surgery are well established including minimal blood loss and short hospital stay. All of our laparoscopic cases were discharged around $6 \mathrm{~h}$ after the procedure. As expected, the hospital stay was longer in the laparotomy group. Despite the simplicity of laparoscopic technique, we found no difference in the operative time. This could be related to the challenge in threading the tapered Mersilene suture into the opening of the suturing device. We recently found that the use of Carter-Thomason CloseSure system facilitates the procedure. The device can be used to pierce the body of the cervix; its tip can be opened like forceps and could grasp the suture easily.

One of the weaknesses of our study is the small sample size. This could be due to our strict indication of the procedure.

\section{Conclusion}

We conclude that abdominal cerclage in women who have failed a transvaginal cervical cerclage is associated with a high live birth rate and it could be performed by laparoscopy.

Conflicts of interest The authors report no conflicts of interest. The authors alone are responsible for the content and writing of the paper.

\section{References}

1. Benson RC, Durfee RB (1965) Transabdominal cervico uterine cerclage during pregnancy for the treatment of cervical incompetency. Obstet Gynecol 25:145-155

2. McDonald I (1957) Suture of the cervix for inevitable miscarriage. J Obstet Gynaecol Br Imp 64:346-353

3. Shirodkar V (1955) A new method of operative treatment of habitual abortions in the second trimester of pregnancy. Antiseptic 52:299-300 
4. Zaveri V, Aghajafari F, Amankwah K, Hannah M (2002) Abdominal versus vaginal cerclage after a failed transvaginal cerclage: a systematic review. Am J Obstet Gynecol 187:868872

5. Al-Fadhli R, Tulandi T (2004) Laparoscopic abdominal cerclage. Obstet Gynecol Clin 31:497-504

6. Whittle WL, Singh SS, Allen L, Glaude L, Thomas J, Windrim R, Leyland N (2009) Laparoscopic cervico-isthmic cerclage: surgical technique and obstetric outcomes. Am J Obstet Gynecol 201:364. e1-364.e7

7. Agdi M, Tulandi T (2008) Placement and removal of abdominal cerclage by laparoscopy. Reprod Biomed Online $16: 308-310$

8. Groom KM, Bryony AJ, Edmonds DK, Benett PR (2004) Preconception transabdominal cervicoisthmic cerclage. Am J Obstet Gynecol 191:230-234 